\title{
Spatial analysis of the relationship between early childhood mortality and malaria endemicity in Malawi
}

\author{
Lawrence N. Kazembe ${ }^{1,2}$, Christopher C. Appleton ${ }^{3}$, Immo Kleinschmidt ${ }^{4}$ \\ ${ }^{1}$ Applied Statistics and Epidemiology Research Unit, Mathematical Sciences Department, Chancellor \\ College, University of Malawi, Zomba, Malawi; ${ }^{2}$ Malaria Research Programme, Medical Research Council, \\ Durban, South Africa; ${ }^{3}$ School of Biological and Conservation Sciences, University of KwaZulu-Natal, \\ Durban, South Africa; ${ }^{4}$ Infectious Diseases Epidemiology Unit, Department of Epidemiology and \\ Population Health, London School of Hygiene and Tropical Medicine, London, UK
}

\begin{abstract}
Spatial differences in mortality have been reported in Africa amongst children under-five years of age. Risk factors contributing to this geographical variation include bio-demographic and socio-economic factors, the prevalence of infectious diseases and the variability in the quality of child health care. This paper is concerned with investigating the link between early childhood mortality and malaria risk. We used data from the Mapping Malaria Risk in Africa (MARA) and Demographic and Health Survey (DHS) databases to explore this relationship. The DHS survey included questions on bio-demographic and socio-economic status, complete birth histories and survival time of each child within the five years preceding the survey. Survival times were computed in months until death or until the survey was done. The malaria risk was based on prevalence data estimated at the precise DHS sampling location. A spatial Cox regression model was applied to analyze child survival, assessing the influence of both individual-specific factors, malaria endemicity and group-specific environmental factors, approximated by geographical location. Geographical location was considered at subdistrict level. Our analysis shows that although malaria endemicity is not associated with the risk of infant mortality, it is an important risk factor for child mortality. The results confirm the effects of bio-demographic and socio-economic variables (maternal education, maternal age, birth order and place of residence) on infant and child mortality. The subdistrict-specific variation of infant and child mortality shows a rural-urban distinction with a relatively lower risk of mortality in main urban areas.
\end{abstract}

Keywords: childhood mortality, malaria, Malawi, spatial analysis, Bayesian inference.

\section{Introduction}

Malaria is common in Malawi and children $<5$ years of age reportedly suffer a mean of 9.7 malaria episodes a year (Wirima, 1996; NSO, 1997). Not only is this parasite infection the cause of over $40 \%$ of deaths in children under two but also one of the most frequent causes of morbidity

Corresponding author:

Lawrence N. Kazembe

Applied Statistics and Epidemiology Research Unit

Mathematical Sciences Department Chancellor College

University of Malawi

PO Box 280 Zomba, Malawi

Tel. +2658668233/1524 222; Fax +2651524046

E-mail: 1kazembe@yahoo.com and mortality in all Malawian children $<5$ years of age. The annual cost of malaria is estimated at US\$ 35 per household, i.e. $7.2 \%$ of the average household income (Ettling et al., 1994).

Spatial differences have been reported in Africa in mortality amongst children under-five years of age (Balk et al., 2004; Gemperli et al., 2004; Kandala and Ghilagaber, 2006). Risk factors contributing to the geographical variation include bio-demographic and socio-economic factors, the prevalence of infectious diseases and the variability in the quality of child health care (Madise and Diamond, 1995; Root, 1999). In addition to the direct link between malaria and early childhood death (Smith et al., 2004; Snow et al., 2004), there are also indirect 
associations through low birth weight and/or anaemia (Kalanda et al., 2005). Malaria and HIV co-exist in much of sub-Saharan Africa and is also common in Malawi. The HIV infection destroys CD4 cells, the immune cells that are required for the development of antimalarial immunity, and almost doubles the risk of malaria parasitaemia and clinical malaria (Taylor and Hoffman, 2000; Abu-Raddad et al., 2006). HIV infection is therefore a major confounder when considering the influence of malaria on child mortality rates.

This paper is concerned with investigating the link between early childhood mortality and malaria. Efforts to quantify the impact of malaria on childhood mortality have been hampered by lack of high quality cause-specific data. Addressing this challenge we used data from the Mapping Malaria Risk in Africa (MARA) and Demographic and Health Survey (DHS) databases to explore the relationship between malaria and childhood mortality. The DHS survey included questions on bio-demographic and socio-economic status, complete birth histories and survival time of each child within the five years preceding the survey. Survival times were computed in months until death or until the survey was carried out. The malaria risk was based on available prevalence data.

Studies on malaria and child mortality in Malawi are few and limited either to hospitals or to small rural areas (Bloland et al., 1996). These do not provide a comprehensive representation of the true situation as a vast number of children die outside the health care system (Government of Malawi, 2002). Moreover, they do not directly assess the role of malaria risk. In this study, the DHS and MARA databases provide an opportunity to explore this relationship in detail.

A spatial Cox regression model was applied (Hennerfeind et al., 2006) to analyze child survival assessing the influence of both individual-specific factors, malaria endemicity and group-specific environmental factors, approximated by geographical location. Geographical location was considered at the subdistrict level using this unit for spatial analy- sis. This choice was driven by the need to highlight small area variation which should not only be more meaningful for health policy decision makers but also improve our understanding of the epidemiology of early childhood mortality in the country. This information is critical for designing interventions to achieve the greatest impact.

Bayesian inference was used to estimate the model through Markov chain Monte Carlo (MCMC) simulation techniques. Three models, i.e. (i) non-spatial, all variables and unstructured random effects; (ii) non-spatial and spatial random effects and all variables; and (iii) spatial and non-spatial effects and all variables without the effect of malaria, were fitted to investigate whether malaria endemicity, socio-economic and bio-demographic covariates explain infant and child mortality.

\section{Materials and methods}

\section{Data}

The data came from the Malawi DHS of the year 2000 (NSO and ORC Macro, 2001), which employed a two-stage stratified sampling design to provide estimates of health and demographic indicators. In the first stage, 560 enumeration areas (EAs), as defined in the Malawi Population and Housing Census of 1998, were selected as primary sampling units, stratified by urban/rural status and with the sampling probability proportional to the population of the selected EA. In the second stage, a fixed number of households were randomly selected in each EA. All women aged 15-49 years were eligible for interview. A total of 13,220 women were interviewed using an interviewer-administered questionnaire which included questions covering complete birth histories.

This study analyzed child survival within the five years preceding the survey. The survival time of each child was computed in months until death or the censoring time (i.e. when the survey was conducted). Individual-specific risk factors, collected as part of Malawi DHS, included socio-economic 
Table 1. Summary of infant and child mortality by selected risk factors included in the analysis.

\begin{tabular}{|c|c|c|c|c|}
\hline \multirow[t]{2}{*}{ Covariates } & \multicolumn{2}{|l|}{ Infant deaths } & \multicolumn{2}{|c|}{ Childhood deaths } \\
\hline & $(\mathrm{n}=1107) \ddagger$ & $\%$ & $(\mathrm{n}=452)$ & $\%$ \\
\hline \multicolumn{5}{|l|}{ Malaria endemicity } \\
\hline $0-25 \%$ & 56 & 9.2 & 18 & 3.0 \\
\hline $25-50 \%$ & 469 & 9.3 & 154 & 3.0 \\
\hline $50-75 \%$ & 479 & 9.2 & 234 & 4.5 \\
\hline $75-100 \%$ & 130 & 9.8 & 46 & 4.4 \\
\hline \multicolumn{5}{|l|}{ Sex } \\
\hline Female & 524 & 8.8 & 220 & 3.9 \\
\hline Male & 583 & 9.8 & 232 & 3.7 \\
\hline \multicolumn{5}{|l|}{ Residence } \\
\hline Urban & 130 & 6.2 & 63 & 3.0 \\
\hline Rural & 977 & 9.9 & 389 & 4.0 \\
\hline \multicolumn{5}{|l|}{ Mother's education } \\
\hline None & 325 & 8.8 & 156 & 4.2 \\
\hline Primary & 736 & 10.0 & 278 & 3.8 \\
\hline Secondary or higher & 46 & 5.5 & 18 & 2.1 \\
\hline \multicolumn{5}{|l|}{ Birth order } \\
\hline 1st born & 347 & 12.0 & 112 & 3.9 \\
\hline 2nd and above & 760 & 8.4 & 340 & 3.8 \\
\hline \multicolumn{5}{|l|}{ Preceding birth interval } \\
\hline$<2$ years & 303 & 13.8 & 122 & 4.9 \\
\hline 2 years or more & 805 & 6.8 & 329 & 3.5 \\
\hline \multicolumn{5}{|l|}{ Mother's age } \\
\hline$<20$ years & 104 & 31.9 & 22 & 5.7 \\
\hline $20-24$ years & 383 & 24.9 & 131 & 4.4 \\
\hline $25-29$ years & 299 & 22.0 & 165 & 4.6 \\
\hline$\geq 30$ years & 321 & 22.6 & 134 & 4.1 \\
\hline
\end{tabular}

‡ The number and proportion who died in each risk category.

and bio-demographic factors (Table 1). Group-specific risk factors included malaria endemicity and environmental factors approximated by geographical location at the subdistrict level. Malaria endemicity was measured by the malaria prevalence rate predicted at the EA level using a geostatistical model developed by Kazembe et al. (2006). Each child was assumed to be equally exposed to malaria risk in each community (EA), with each child in the same EA assigned the same risk value. The malaria data were from children of age $\leq 10$ years, collected retrospectively at 73 different sites, and are consistent with the mortality data used here. The prevalence rates were categorized into four sub-groups: low (0-25\%), medium $(25-50 \%)$, high $(50-75 \%)$ and very high $(75-100 \%)$ levels of malaria endemicity.

Table 1 presents descriptive summaries of the mortality rates by covariates. The risk for both groups varied with demographic and socio-economic factors. Within the five years of the survey, $13 \%$ of children $(n=1559)$ had died. Of these, 1107 $(77 \%)$ died during the first 12 months of their life, and the remaining $452(23 \%)$ died between their first and fifth birthday. The mean rate was 150 child deaths per 1000. The mean survival time for infants was 4 months (median $=3$ months), whereas the mean child survival time was 23 months (median = 24 months).

\section{Statistical modeling}

A spatial Cox regression model (Hennerfeind et al., 2006) was applied to determine factors associated with the risk of early childhood mortality. Assuming that $T_{i j}$ is the observed number of months lived or the censoring time for $j$-th child in area $i$, the hazard function at time $T=t$ is given by the equation 


$$
h\left(\mathrm{t} \mid \beta, v_{i j}\right)=\mathrm{h}_{0}(t) \exp \left(\beta v_{i j}\right)
$$

where $\mathrm{h}_{0}(t)$ is the baseline hazard at time $t$, and the $\beta$ s are a vector of regression coefficients for the fixed and time-invariant variables $\left(v_{i j}\right)$. The exponent of a coefficient, i.e. $\exp (\beta)$, is interpreted as the hazard ratio $(\mathrm{HR})$, i.e. the ratio of instantaneous risk which is assumed to be constant over time. Since individuals were clustered in geographical regions, a group-specific random frailty term, $\Psi_{i}$, was introduced to augment the Cox model, i.e.

$$
h\left(\mathrm{t} \mid \beta, v_{i j}\right)=\mathrm{h}_{0}(t) \exp \left(\beta v_{i j}+\Psi_{i}\right)
$$

The above model indicates that childhood survival is influenced by both individual-specific factors $\left(v_{i j}\right)$ and group-specific environmental factors $\Psi_{i}$. Here it is assumed that the environmental factors are approximated by geographical location. Two spatial structures are distinguished:

(i) spatially distributed random effects, $u_{i}$ (to capture similarities across areas), and

(ii) unstructured random effects, $u_{i}$ (to allow within-area heterogeneity) so that $\Psi_{i}=s_{i}+v_{i}$ (Besag et al., 1991).

Fitting the model above (2) assumes a semi-parametric additive predictor, known as the geoadditive survival model (Hennerfeind et al., 2006), which results in

$$
\eta_{i j}(t)=f_{0}(t)+\beta v_{i j}+u_{i}+s_{i}
$$

where $\eta_{i j}$ is the log-additive predictor at time $t$ for child $j$ in area $i$. The term $f_{0}(t)=\log \left(h_{0}(t)\right)$ is the $\log$ baseline hazard effect at time $t$. The other terms are as defined above.

A Bayesian approach was used to estimate the model above (3) with the following prior distributions specified for the parameters. The spatially structured component $\left(s_{i}\right)$ was assigned an intrinsic conditional autoregressive (CAR) prior (Besag et al., 1991). This assumes that the mean for each area, $s_{i}$, conditional on the neighbouring areas, has a normal distribution with its mean equal to the average of neighbouring areas, $s_{i}$, and variance inversely proportional to the number of neighbours, $m_{i}$. The CAR prior has the form of

$$
p\left(s_{i} \mid s_{i} ; l \text { neighbouring } i\right) \sim N\left(\frac{1}{m_{i}} \sum_{\text {ladj } i} s i, \frac{\tau_{s}^{2}}{m_{i}}\right)
$$

where $l$ adj $i$ denotes that $i$ and $l$ are adjacent areas and $\tau_{s}^{2}$ is a spatial variance, which at a further step of hierarchy, is modeled using inverse gamma (IG) with known parameters, i.e. $a=1, b=0.005$. The unstructured random effects, $u_{i}$, were assumed to follow an exchangeable normal prior, $u_{i} \sim N\left(0, \tau_{u}^{2}\right)$, where $\tau_{u}^{2}$ measures the degree of heterogeneity, which again was assigned an IG hyper prior. The baseline hazard effect, $f_{0}(t)$, was assigned a penalized spline with a second-order random-walk prior. The fixed regression coefficients were assigned diffuse priors. Inference was based on samples drawn from the posterior distribution using MCMC techniques. Model fit was implemented in BayesX 1.4 (Brezger et al., 2005). For all models, 25,000 iterations were run with the initial 5000 discarded and every $20^{\text {th }}$ sample stored to give a final sample of 1000 for parameter estimation.

For infant and child mortality, three models were fitted to investigate whether malaria endemicity, socio-economic and bio-demographic covariates can explain the rates. The first model (M1) was nonspatial and included all variables and the unstructured random effects to allow for sample design. Model M2 included spatial and non-spatial random effects and all variables, while model M3 also included spatial and non-spatial random effects and all variables except the effect of malaria. The model comparison was based on the deviance information criterion (DIC) (Spiegelhalter et al., 2002) given by the equation

$$
D I C=\bar{D}+p_{D}
$$

where $\bar{D}$ is the deviance of the model evaluated at the posterior mean of the parameters, and represents the fit of the model to the data. The component $p_{D}$ is the effective number of parameters assess- 
Table 2. Fixed and random estimates of the three models fitted on infant mortality (1q0) in Malawi.

\begin{tabular}{|c|c|c|c|}
\hline \multirow[t]{2}{*}{ Covariates } & Model 1‡ & Model 2 & Model 3 \\
\hline & $\operatorname{HR}(95 \% \mathrm{CI})$ & $\operatorname{HR}(95 \% \mathrm{CI})$ & $\operatorname{HR}(95 \% \mathrm{CI})$ \\
\hline \multicolumn{4}{|l|}{ Malaria endemicity } \\
\hline $0-25 \%$ & 1.00 & 1.00 & \\
\hline $25-50 \%$ & $1.01(0.88,1.18)$ & $0.99 \quad(0.87,1.16)$ & \\
\hline $50-75 \%$ & $0.98(0.86,1.14)$ & $0.96(0.85,1.12)$ & \\
\hline $75-100 \%$ & $1.04(0.86,1.27)$ & $1.12(0.90,1.35)$ & \\
\hline \multicolumn{4}{|l|}{ Sex } \\
\hline Female & 1.00 & 1.00 & 1.00 \\
\hline Male & $1.07(1.01,1.13)$ & $1.07(1.01,1.14)$ & $1.07(1.01,1.14)$ \\
\hline \multicolumn{4}{|l|}{ Residence } \\
\hline Rural & 1.00 & 1.00 & 1.00 \\
\hline Urban & $0.79(0.70,0.88)$ & $0.78 \quad(0.70,0.88)$ & $0.77 \quad(0.69,0.88)$ \\
\hline \multicolumn{4}{|l|}{ Mother's education } \\
\hline None & 1.00 & 1.00 & 1.00 \\
\hline Primary & $1.26(1.12,1.42)$ & $1.26(1.13,1.42)$ & $1.26(1.13,1.41)$ \\
\hline Secondary or higher & $0.71 \quad(0.57,0.87)$ & $0.72(0.58,0.86)$ & $0.71 \quad(0.57,0.86)$ \\
\hline \multicolumn{4}{|l|}{ Birth order } \\
\hline 1st born & $1.83(1.42,2.32)$ & $1.80(1.44,2.29)$ & $1.83(1.43,2.32)$ \\
\hline 2nd and above & 1.00 & 1.00 & 1.00 \\
\hline \multicolumn{4}{|l|}{ Preceding birth interval } \\
\hline$<2$ years & $1.61(1.42,1.83)$ & $1.62(1.42,1.79)$ & $1.63(1.43,1.81)$ \\
\hline 2 years or more & 1.00 & 1.00 & 1.00 \\
\hline \multicolumn{4}{|l|}{ Mother's age } \\
\hline$<20$ years & $1.04(0.70,1.55)$ & $1.04 \quad(0.71,1.52)$ & $1.05 \quad(0.72,1.49)$ \\
\hline $20-24$ years & $1.18(0.94,1.49)$ & $1.18 \quad(0.94,1.49)$ & $1.77(0.92,1.45)$ \\
\hline $25-29$ years & $0.97(0.77,1.20)$ & $0.98 \quad(0.78,0.13)$ & $0.97(0.78,1.22)$ \\
\hline$\geq 30$ years & 1.00 & 1.00 & 1.00 \\
\hline \multicolumn{4}{|l|}{ Variance components } \\
\hline$\tau_{u}^{2}$ & $0.10 \quad(0.04,0.18)$ & $0.05(0.004,0.13)$ & $0.01(0.001,0.10)$ \\
\hline$\tau_{s}^{2}$ & & $0.09 \quad(0.01,0.22)$ & $0.16 \quad(0.03,0.37)$ \\
\hline$\phi$ & & 0.57 & 0.80 \\
\hline \multicolumn{4}{|l|}{ Model fit } \\
\hline $\bar{D}$ & 7057.34 & 7077.05 & 7098.22 \\
\hline$p_{D}$ & 74.82 & 65.24 & 53.13 \\
\hline DIC & 7206.98 & 7207.54 & 7204.48 \\
\hline
\end{tabular}

Legend: $\mathrm{HR}=$ hazard ratio, $\mathrm{CI}=$ credible interval; DIC = deviance information criterion (see text). $¥$ Model 1 is non-spatial with all variables including malaria and unstructured random effects; model 2 is spatial with all variables; model 3 is also spatial, but excluded the effects of malaria.

ing the complexity of the model. Since small values of $\bar{D}$ indicate a good fit, while small values of $p_{D}$ point in the direction of a parsimonious model, small DIC values reflect a better model.

The proportion of total residual spatial variance was computed as $\phi=\boldsymbol{\tau}_{s}^{2} /\left(\boldsymbol{\tau}_{s}^{2}+\boldsymbol{\tau}_{u}^{2}\right)$. The closer this ratio is to 1 , the more dominant is the spatially structured component, while the closer it is to 0 , the more dominant is the unstructured random effect. When $\phi$ is close to 0.5 , it is not possible to distinguish between the two outcomes. In this case, the total variance $\left(\boldsymbol{\tau}_{s}^{2}+\boldsymbol{\tau}_{u}^{2}\right)$ can be plotted.

\section{Results}

Table 2 presents results for the three models fitted for infant mortality. Model M3 did not include malaria and was the best fit (DIC $=7204.48)$. The risk varied with demographic and socio-economic factors. The risk was higher for boys than for girls and rural children were more at risk than urban children. The level of risk was inversely proportion- 
al to the level of education of the mothers, i.e. children of mothers with high-school education were less at risk than children born to mothers with only primary school education, or to mothers without any education. In addition, first-born children were at increased risk and birth interval if less than two years also placed children at increased risk.

Figure 1A shows the plot of residual spatial effects based on model M3 representing unobserved, or unknown, environmental or geographical factors. A clear spatial variation is displayed in the map with increased hazard along the lakeshore regions and in the southern region and decreased hazard in the central-western areas and in the north of the country (confirmed by the standard errors in Figure 1B). Spatially structured variation also overwhelmed the unstructured spatial heterogeneity $(\phi=0.8)$.

The results for child mortality are given in Table 3. Based on the DIC, model M2 showed the best fit $(\mathrm{DIC}=3825.36)$. The risk of child mortality increased in areas with $50-75 \%$ malaria endemicity. Rural children were at slightly higher risk compared
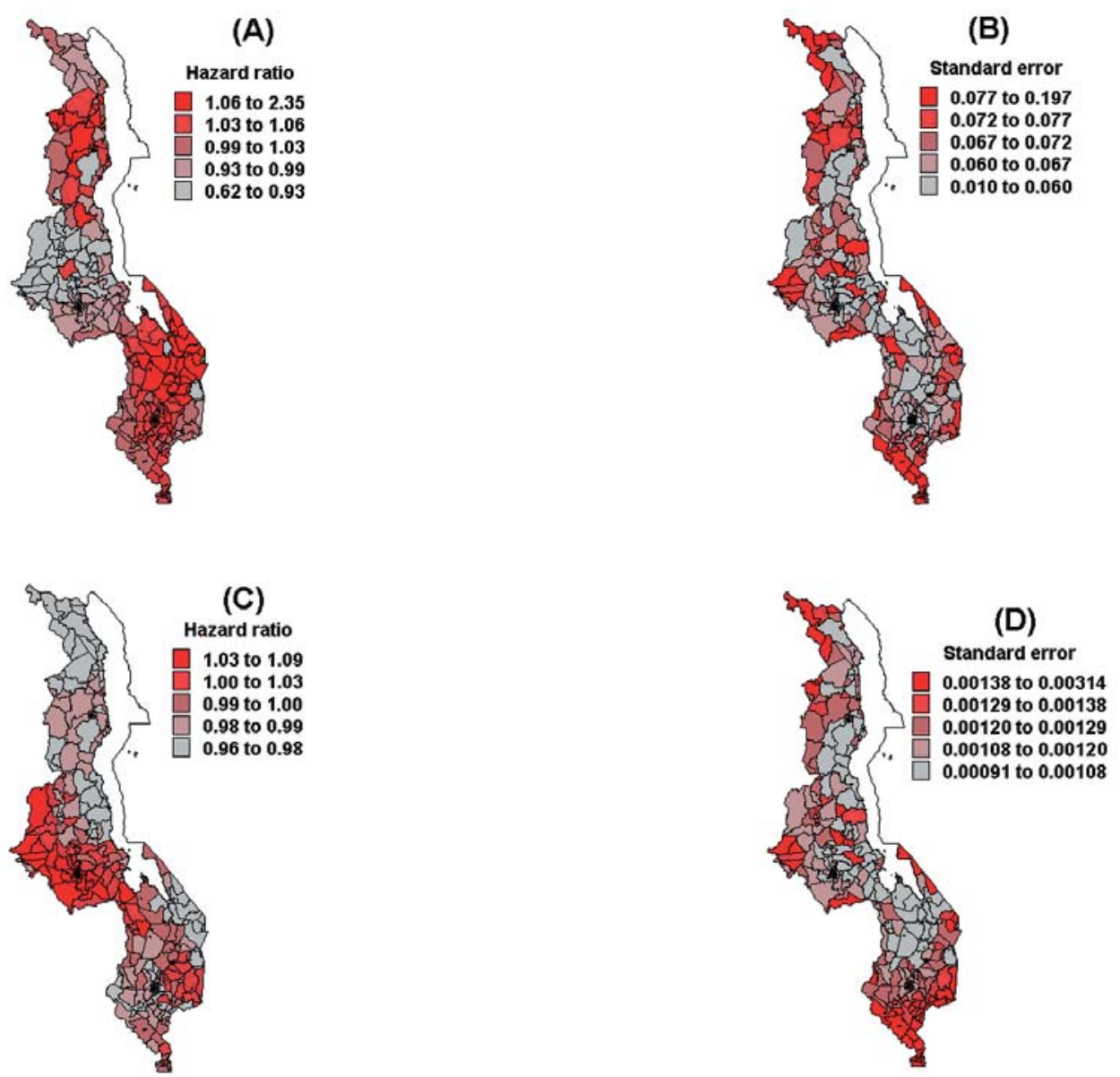

Fig. 1. Maps showing residual spatial effects (subdistrict hazard ratios) and the corresponding standard errors for infant and child mortality. Map (A) shows the residual spatial effects for the infant mortality model and the corresponding standard errors (map (B)) based on model M3 in Table 2. Map (C) shows residual spatial effects for the child mortality model and the corresponding standard errors (map (D)) based on M2 in Table 3. 
Table 3. Fixed and random estimates of the three models fitted on child mortality (4q1) in Malawi.

\begin{tabular}{|c|c|c|c|}
\hline \multirow[t]{2}{*}{ Covariates } & Model 1‡ & Model 2 & Model 3 \\
\hline & $\operatorname{HR}(95 \% \mathrm{CI})$ & $\operatorname{HR}(95 \% \mathrm{CI})$ & $\operatorname{HR}(95 \% \mathrm{CI})$ \\
\hline \multicolumn{4}{|l|}{ Malaria endemicity } \\
\hline $0-25 \%$ & 1.00 & 1.00 & \\
\hline $25-50 \%$ & $0.84 \quad(0.68,1.01)$ & $0.82(0.67,1.01)$ & \\
\hline $50-75 \%$ & $1.26(1.05,1.51)$ & $1.26(1.04,1.54)$ & \\
\hline $75-100 \%$ & $1.28(0.99,1.62)$ & $1.25(0.97,1.58)$ & \\
\hline \multicolumn{4}{|l|}{ Sex } \\
\hline Female & 1.00 & 1.00 & 1.00 \\
\hline Male & $1.03(0.94,1.12)$ & $1.03(0.95,1.13)$ & $1.03(0.94,1.14)$ \\
\hline \multicolumn{4}{|l|}{ Residence } \\
\hline Rural & 1.00 & 1.00 & 1.00 \\
\hline Urban & $0.91 \quad(0.79,1.00)$ & $0.91 \quad(0.80,1.00)$ & $0.92 \quad(0.80,1.01)$ \\
\hline \multicolumn{4}{|l|}{ Mother's education } \\
\hline None & 1.00 & 1.00 & 1.00 \\
\hline Primary & $1.18(0.99,1.42)$ & $1.18 \quad(0.97,1.44)$ & $1.17 \quad(0.96,1.46)$ \\
\hline Secondary or higher & $0.64 \quad(0.46,0.84)$ & $0.62(0.45,0.86)$ & $0.62(0.41,0.86)$ \\
\hline \multicolumn{4}{|l|}{ Birth order } \\
\hline 1st born & $1.34 \quad(0.93,1.88)$ & $1.39(0.94,2.01)$ & $1.35 \quad(0.93,1.98)$ \\
\hline 2nd and above & 1.00 & 1.00 & 1.00 \\
\hline \multicolumn{4}{|l|}{ Preceding birth interval } \\
\hline$<2$ years & $1.26(1.03,1.52)$ & $1.29(1.06,1.54)$ & $1.29(1.06,1.56)$ \\
\hline 2 years or more & 1.00 & 1.00 & 1.00 \\
\hline \multicolumn{4}{|l|}{ Mother's age } \\
\hline$<20$ years & $2.23(1.22,3.99)$ & $2.22(1.14,3.92)$ & $2.26(1.14,3.99)$ \\
\hline $20-24$ years & $1.24 \quad(0.92,1.70)$ & $1.24(0.90,1.75)$ & $1.24 \quad(0.90,1.75)$ \\
\hline $25-29$ years & $1.43(1.05,1.95)$ & $1.43(1.06,1.93)$ & $1.42(1.05,1.89)$ \\
\hline$\geq 30$ years & 1.00 & 1.00 & 1.00 \\
\hline \multicolumn{4}{|l|}{ Variance components } \\
\hline$\tau_{u}^{2}$ & $0.02(0.001,0.09)$ & $0.01(0.001,0.07)$ & $0.01(0.001,0.05)$ \\
\hline$\tau_{s}^{2}$ & & $0.004(0.001,0.01)$ & $0.16 \quad(0.02,0.43)$ \\
\hline$\phi$ & & 0.4 & 0.80 \\
\hline \multicolumn{4}{|l|}{ Model fit } \\
\hline $\bar{D}$ & 3780.59 & 3787.85 & 3762.24 \\
\hline$p_{D}$ & 23.98 & 18.76 & 35.36 \\
\hline DIC & 3828.55 & 3825.36 & 3832.96 \\
\hline
\end{tabular}

Legend: $\mathrm{HR}=$ hazard ratio, $\mathrm{CI}=$ credible interval; DIC = deviance information criterion (see text). $¥$ Model 1 is non-spatial with all variables including malaria and unstructured random effects; model 2 is spatial with all variables; model 3 is also spatial, but excluded the effects of malaria.

to urban children. Having mothers with secondary or higher education in contrast to no or primary education provided relative protection. Children were at greater risk if the preceding birth interval was less than two years and if the mother was under 20 or between 25-29 years of age relative to mothers older than that. Significant subdistrict-specific residual risk was evident, as depicted by the total residual effects, i.e. $\exp (u) \cdot \exp (s)$ (Figure 1C), and confirmed by the standard errors (Figure 1D). Relatively high risk was observed in the central and southern region, while low risk prevailed in parts of the northern and southern regions.

\section{Discussion}

This study is one of the few assessing the spatial pattern of early childhood mortality and the effect of malaria endemicity. Apart from Gemperli et al. (2004), for example, studies have generally focused on the role of socio-economic and bio-demographic covariates. Importantly, these have been included in 
this analysis as confounding covariates. In agreement with previous studies (Madise and Diamond, 1995; Root, 1999; Balk et al., 2004; Gemperli et al., 2004; Kandala and Ghilagaber, 2006), the present results confirm the link between socio-economic and demographic factors (such as place of residence, maternal age, maternal education, birth spacing and birth order) and the risk of childhood mortality in Malawi. On one hand, our present analysis shows that malaria endemicity, in addition to the effect of socio-economic and bio-demographic factors, is associated with early childhood mortality. On the other, this effect was shown to be different when infants and children were compared. Indeed, the risk of infant mortality is similar at all levels of malaria endemicity, despite the bivariate data showing that the risk of infant mortality marginally increased at the highest level of malaria endemicity (category $75-100 \%$ in Table 1 ). For child mortality, however, the malaria risk was found to impose a greater risk at the intermediate or higher levels ( $\geq 50 \%$ prevalence) than at lower levels of endemicity. Certainly, this effect was evident from the bivariate data (Table 1), although not strongly significant in Model 2 of Table 3. However, category 25-50\% was associated with similar risk of deaths relative to the low $(0-25 \%)$ category.

The differential impact of malaria risk can be explained from two perspectives. For infants, it is likely that the effect of maternal immunity is still protective in the first six months of life (Smith et al., 2004; Snow et al., 2004). Nevertheless, the indirect consequences of malaria transmission intensity on infant mortality cannot be ruled out. Research in this area indicates that the incidence of low birth weight is an indirect attribute of maternal malaria which acts on the infant at the foetal stage of life (Steketee et al., 2001). In endemic areas, maternal malaria infection is known to predispose towards pre-term delivery, which in turn leads to peri- or neonatal mortality (Van Geertruyden et al., 2004). The impact of maternal malaria is reported to be greater among primigravidae than in multigravidae women (Steketee et al., 2001). Furthermore, mater- nal malaria is associated with retarded early growth and under-nutrition (Kalanda et al., 2005), which mostly manifests in infants at the age of 6-12 months. Under-nutrition and retarded growth are the underlying causes of more than $53 \%$ of all cases of child death that occur annually (Caulfield et al., 2004).

In general, the risks an infant faces during birth and the first 11 months of life are very different from those experienced after this period. Infant deaths, especially at the neonatal stage, are more closely linked to endogenous factors such as congenital malformations, hereditary diseases and low birth weight (Balk et al., 2004). Older children are more likely to die of exogenous factors such as accidents and malnutrition, while malaria and other preventable infectious diseases present an increased impact due to the waning of maternal immunity (Van Geertruyden et al, 2004). However, this distinction may not always be true. For example, high levels of HIV in children attributed to mother-tochild transmission strongly contributes to childhood mortality, particularly in the first two years of life (Taylor et al., 2000).

The subdistrict-specific geographical variation in infant and child mortality showed consistently higher risk in rural than in urban settings (Fig. 1). This urban-rural distinction could be a combined result of many factors. Malaria transmission is generally more intense in rural areas even though the malaria prevalence map (Kazembe et al., 2006) is not sufficiently detailed to show this. Rural children, regardless of stage of life, suffer higher mortality as a result of increased exposure to Plasmodium falciparum, which our malaria variable only partly explains. Rural areas are also the most deprived, for instance due to severe and deep poverty, non-availability or inaccessibility of prompt health care. In addition, other shared community factors might impose largescale clustering of putative factors, hence the spatial variation in the health outcome (Benson et al., 2005). Unobserved risk of common and leading diseases of childhood mortality such as diarrhoea, measles, HIV/AIDS and acute respiratory infections 
(e.g. pneumonia), some of which vary spatially (Kandala et al., 2006), may induce a disproportionate patterning in child mortality with poor or rural areas experiencing increased hazards (Benson et al., 2005). Taken together, the burden of childhood mortality may be higher in rural areas than in most urban areas.

The linkage between the DHS mortality data and the estimated malaria prevalence data provides an exciting analysis based on the epidemiological evidence that endemic malaria increases the risk of early childhood mortality. The implicit basic assumption in these analyses is that the frequency or duration of exposure to malaria, coupled with other individual factors (e.g. age, location and behavioural factors) influences early childhood mortality. The fundamental problem is whether cross-sectional data are sufficient to confirm this kind of hazard-exposure linkage. In our opinion, the data obtained and presented in this study provide a possible connection between hazard and exposure (Gemperli et al., 2004).

Since our results depend on the reliability of the survival times, the limitations of this study hinges primarily on the potential shortcoming of using mothers' reports on childhood mortality. This may introduce a bias with regard to survival times as outcomes are often imputed and lumped due to rounding of age. Nevertheless, many studies using DHS data have reported reliable results even from the least educated women in rural areas (Balk et al., 2004; Gemperli et al., 2004). Another limitation is the possibility of measurement errors of the estimated malaria values, which may bias the results. This approach has been used successfully by others, e.g. Gemperli et al. (2004), and is likely to give a conservative estimate of malaria risk at DHS sampling locations.

Another limitation of this study is the lack of data on HIV prevalence, particularly since the experience in sub-Saharan Africa suggests that this infection is an important cause of reported child mortality. Lack of effective treatment programmes using anti-retroviral drugs to prevent mother-to-child transmission is likely to result in increased HIV transmission. The viral load of HIV of the mother also affects HIV transmission and the influence of HIV may therefore cause infant and child mortality. It is currently estimated that $50 \%$ of untreated, HIV-positive children die before the age of two years and that another 30\% do not make it past five (Taylor et al., 2000; Abu-Raddad et al., 2006). HIV infection is thus a major confounder when considering the influence of malaria on child mortality rates, thus, these studies might be strengthened by controlling for HIV prevalence. This modeling extension can be the focus of further study, when HIV data become available at child level.

Despite the limitations discussed above, we feel that this study fills a significant gap in the knowledge of geographical variations of early childhood mortality in Malawi. The maps identify areas of increased hazard and these patterns have important implications for health policy aimed at reducing allcause child mortality by $50 \%$ by 2010 . Such a policy may take a comprehensive approach aiming at:

(i) a reduction in HIV incidence which can affect malaria immunity;

(ii) targeted prevention of mother-to-child treatment programmes;

(iii) integrated management of childhood illnesses (IMCI); and

(iv) dedicated malaria control programmes designed for areas where high childhood mortality have been found.

\section{Acknowledgements}

LNK would like to acknowledge the research training grant received from WHO/TDR and support from the Medical Research Council, Durban, South Africa during his PhD training. We also acknowledge permission granted by MEASURE DHS to use the 2000 Malawi DHS data under the project "Spatial analysis of malariometric indicators in Malawi".

\section{References}

Abu-Raddad LJ, Patnail P, Kublin JG, 2006. Dual infection with HIV and malaria fuels the spread of both diseases in sub-Saharan Africa. Science 314,1603-1606. 
Balk D, Pullum T, Storeygard A, Neuman M, 2004. A spatial analysis of childhood mortality in West Africa. Pop Space Place 10, 175-216.

Benson T, Chamberlin J, Rhinehart I, 2005. An investigation of the spatial determinants of the local prevalence of poverty in rural Malawi. Food Policy 30, 532-550.

Besag J, York J, Mollie A, 1991. Bayesian image restoration with two applications in spatial statistics (with discussion). Ann Instit Stat Math 43, 1-59.

Bloland P, Slutsker L, Steketee RW, Wirima JJ, Heymann DL, Breman JG, 1996. Rates and risk factors for mortality during the first two years of life in rural Malawi. Am J Trop Med Hyg 55, 82-86.

Brezger A, Kneib T, Lang S, 2005. BayesX: analysing Bayesian structured additive regression models. J Stat Soft 14, 11.

Caulfield LE, de Onis M, Lossner M, Black RE, 2004. Undernutrition as an underlying cause of child deaths associated with diarrhoea, pneumonia, malaria, and measles. Am J Clin Nutr 80, 193-198.

Ettling M, Steketee RW, Macheso A, Schultz LJ, Nyasulu Y, Chitsulo L, 1994. Malaria knowledge, attitudes and practices in Malawi: survey population characteristics. Trop Med Parasitol 45, 57-60.

Gemperli A, Vounatsou P, Kleinschmidt I, Bagayoko M, Lengeler C, Smith T, 2004. Spatial patterns of infant mortality: effects of malaria endemicity. Am J Epidemiol 159, 64-72.

Government of Malawi (GoM), 2002. Malaria Policy. National Malaria Control Programme. Community Health Sciences Unit. Lilogwe: Government of Malawi, 69 pp.

Hennerfeind A, Brezger A, Fahrmeir L, 2006. Geoadditive survival model. J Am Stat Ass 101, 1065-1075.

Kalanda BF, van Buuren S, Verhoeff FH, Brabin BJ, 2005. Catch-up growth in Malawian babies, a longitudinal study of normal and low birthweight babies born in a malarious endemic area. Early Hum Dev 81, 841-850.

Kandala N-B, Ghilagaber G, 2006. A geo-additive Bayesian discrete-time survival model and its application to spatial analysis of childhood mortality in Malawi. Qual Quant 40, 935-957.

Kandala N-B, Magadi MA, Madise NJ, 2006. An investiga- tion of district spatial variations of childhood diarrhoea and fever morbidity in Malawi. Soc Sci Med 62, 1138-1152.

Kazembe LN, Kleinschmidt I, Holtz TH, Sharp BL, 2006. Spatial analysis and mapping of malaria risk in Malawi using point referenced prevalence of infection data. Int $\mathrm{J}$ Health Geogr 5, 41.

Madise NJ, Diamond I, 1995. Determinants of infant mortality in Malawi: an analysis to control for death clustering within families. J Biosoc Sci 27, 25-106.

National Statistical Office (NSO), 1997. Knowledge, attitudes and practices in health survey 1996. NSO: Zomba, Malawi, 281 pp.

National Statistical Office (NSO), ORC Macro, 2001. Malawi demographic and health survey 2000. NSO, Zomba, Malawi, 384pp. Available online at [http://www.measuredhs.com] and [http://nso.malawi.net].

Root G, 1999. Disease environments and subnational patterns of under-five mortality in sub-Saharan Africa. Int J Pop Geogr 5, 117-132.

Smith TA, Leuenberger R, Lengeler C, 2004. Child mortality and malaria transmission intensity in Africa. Trends Parasitol 17, 145-149.

Snow RW, Korenromp EL, Gouws E, 2004. Paediatric mortality in Africa: P. falciparum malaria as a cause or risk? Am J Trop Med Hyg 71, 16-24.

Spiegelhalter DJ, Best NG, Carlin BP, van der Linde A, 2002. Bayesian measures of model complexity and fit (with discussion). J R Stat Soc B 64, 1-34.

Steketee RW, Nahlen BL, Parise ME, Menendez C, 2001. The burden of malaria in pregnancy in malaria-endemic countries. Am J Trop Med Hyg 64, 28-35.

Taylor TE, Hoffman IF, 2000. Can HIV-1 infections in Africa provide insights into acquired immunity to malaria? Lancet 356, 1046.

Van Geertruyden J-P, Thomas F, Erhart A, D'Alessandro U, 2004. The contribution of malaria in pregnancy to perinatal mortality. Am J Trop Med Hyg 71, 35-40.

Wirima JJ, 1996. A nation-wide malaria knowledge, attitudes and practices survey in Malawi. Trop Med Parasitol $45,52-53$. 